\title{
In silico clinical trials: how computer simulation will transform the biomedical industry
}

\author{
Marco Viceconti ${ }^{1}$, Adriano Henney ${ }^{2}$, and Edwin Morley-Fletcher ${ }^{3}$ \\ ${ }^{1}$ Department of Mechanical Engineering and INSIGNEO Institute for In silico Medicine, University of Sheffield, \\ United Kingdom \\ ${ }^{2} \mathrm{VPH}$ Institute for Integrative Biomedical Research \\ ${ }^{3}$ Linkeus srl
}

Received: 08 February2015

Accepted: 16 April 2016

*Correspondence:

Marco Viceconti,

E-mail: m.viceconti@sheffield.ac.uk

Copyright: (c) the author(s), publisher and licensee Medip Academy. This is an open-access article distributed under the terms of the Creative Commons Attribution Non-Commercial License, which permits unrestricted non-commercial use, distribution, and reproduction in any medium, provided the original work is properly cited.

\begin{abstract}
The term 'in silico clinical trials indicates the use of individualised computer simulation in the development or regulatory evaluation of a medicinal product, medical device, or medical intervention. This review article summarises the research and technological roadmap developed by the Avicenna Support Action during an 18 month consensus process that involved 577 international experts from academia, the biomedical industry, the simulation industry, the regulatory world, etc. The roadmap documents early examples of in silico clinical trials, identifies relevant use cases for in silico clinical trial technologies over the entire development and assessment cycle for both pharmaceuticals and medical devices, identifies open challenges and barriers to a wider adoption and puts forward 36 recommendations for all relevant stakeholders to consider.
\end{abstract}

Keywords: Computer modelling and simulation, Clinical trials, In silico medicine, Predictive medicine

\section{INTRODUCTION}

Any biomedical product to be distributed commercially must undergo a development and assessment process before being placed on the market. The appropriate level of scrutiny and rigorous testing before commercialization is of paramount importance, due to the risk of potential harm. In most cases, especially for products that fall into the higher risk classes, the producing company must demonstrate the efficacy of the product in healing or alleviating the effects of a disease or disability, as well as an acceptable safety profile, before any widespread use. Today, the only conclusive (and accepted) way to ensure the safety and efficacy of a biomedical product is to test it on living organisms, first on animals (preclinical evaluation) and then on humans (clinical evaluation).
The preclinical evaluation process represents an essential step in the development of any potential biomedical product. It is the means by which the fundamental basis for how a product might work is evaluated and, hopefully, confirmed. However, due to the hugely complex nature of human diseases, the significant differences between individuals, and the inevitable variability in how a treatment is administered, it is not unusual for a product to perform exceptionally well in tightly controlled laboratory studies, but show some serious problems during clinical trials. According to the Tufts Center for the Study of Drug Development, ${ }^{2}$ the development of a new pharmaceutical product, and its introduction into the market, is estimated to exceed US $\$ 2.5$ billion, over $75 \%$ of which is spent on in vivo studies, whether on animals or on humans. Every time a 
product fails late in the process, for example at the end of phase II or even in phase III trials, companies suffer a huge loss.

Whilst clinical trials may tell us that a product is unsafe or ineffective, they rarely tell us why, or suggest how to improve it. As such, a product that fails during clinical trials may simply be abandoned, even if a small modification might solve the problem, resulting in the potential creation of an 'all-or-nothing' mind-set in the biomedical industry.

Such a paradigm stifles innovation, decreasing the number of truly original biomedical products presented to the market every year, and at the same time increasing the cost of development (which, paradoxically, further increases the risk as the pressure to contain such costs increases). As a result, it is also becoming increasingly difficult for companies to undertake the development of new products targeting rare conditions, either because the associated costs cannot be justified against the limited return on investment, or because the resulting sale prices would be so high as to pose a challenge for universal healthcare systems.

The biomedical industry is not the only sector that deals with highly complex and potentially critical systems. In other industrial sectors, such as aerospace or nuclear industries, computer modeling and simulation is used extensively during both product development and assessment to overcome similar safety problems with mission-critical products. Can the same approach be used for biomedical products? In addition to traditional in vitro and in vivo studies, might we adopt a third way for developing and testing biomedical products by making use of this 'in silico' technology?

In 1955, Solomon and Gold published a three compartment model of potassium transport in human erythrocytes. ${ }^{2}$ This appears to be the first paper indexed by Index Medicus (now PubMed) with the keywords physiology and computer. From that first study until the late 1980 s, most computer models aimed to capture the basic mechanisms underlying physiological or pathological processes in mathematical form, without intending to make quantitatively accurate predictions. In the 1990s, the development of stochastic modeling and increased computational powers enabled the development of population-specific models that aimed to predict the average value of specific quantities over a population. ${ }^{3-10}$ In the early 2000 s, the computational ecology community started to debate the virtues of individual-based models for population ecology. ${ }^{11}$ Soon after, in silico medicine research also began to use the first patient-specific models ${ }^{12-17}$ and some analysts started to suggest that such approaches could be useful in the development of new medical products. ${ }^{18}$

In 2007, a group of experts published "seeding the EuroPhysiome: a roadmap to the virtual physiological human". They presented a scenario where imaging and sensing technologies were used to generate quantitative information about the biology, physiology, and pathology of a patient at different scales of space and time. This information would then be used as the input for multiscale computer models encapsulating all the knowledge available for a given disease process, in order to produce patient-specific predictions for diagnosis, prognosis, and treatment planning. Since then, dozens of single research teams and consortia around the world have developed a whole set of new technologies and methods, initiated with a similar perspective to that original research roadmap. While the vision of the Virtual Physiological Human (VPH) is not yet entirely realised, VPH technologies are being assessed clinically in a number of practical applications and preliminary results suggest important improvements over current standards of care. In some of these projects it has been necessary to simulate the treatment in addition to the pathophysiology in order to predict how a patient would respond to a particular treatment option. In the RT3S project, the deployment and the fatigue cycling of peripheral vascular stenting was modeled. ${ }^{19}$ The VPHOP project included a model of the effect of vertebroplasty in adjacent vertebrae. ${ }^{20}$ Some other projects have gone even further, for example, the PreDICT project, which used VPH models to assess the cardio-toxicity of new drugs. ${ }^{21}$ Another project used an in silico acute stroke model to explore why hundreds of compounds that have been shown efficacious in rodent models failed in phase II or III clinical trials: the ratio of astrocytes over neurons, which is quite different in human brains and in rodents, was suggested as the cause. ${ }^{22}$ One of the essential traits of the VPH approach is the recognition that there is no preferential scale, and each problem should be tackled starting from the space-time scale where the process is observed (middle-out approach).

Of course this is not the only approach that was pursued. Many research teams worldwide adopted a bottom-up process, in an attempt to translate the systems biology approach into clinical practice. ${ }^{23-25}$ Some envisaged a future model of Predictive, Preventive, Personalized and Participatory medicine (P4) based on the translation of systems biology, or as it has later become known, systems medicine. ${ }^{26}$ While this approach holds the potential for huge impact, especially in relation to the discovery of new pharmaceutical compounds, in many cases there are knowledge gaps that make the clinical application difficult. ${ }^{27}$ One particularly important limitation is the ability to model cell-tissue interactions, as was stressed in the 2009 workshop jointly organised by the United States Environmental Protection Agency and the European Commission . Some authors have tried to bridge this with phenomenological models, such as the Effect Model Law. ${ }^{28,29}$ All these research activities embraced a scenario in which VPH models could be used not to enhance the clinical management of patients affected by particularly difficult pathologies, but rather to design and assess biomedical products. In 2011, the VPH 
Institute introduced the term 'in silico clinical trials' (ISCT) to describe this type of activity.

The biomedical industry already uses computer modeling and simulation in the development, and to a lesser extent in the assessment process, but such use is currently limited:

a) Medical device companies use it mostly in the design phase and what is modeled is primarily the device (where conventional engineering modeling techniques can be used), whereas the host organism is mostly reduced to some boundary / environmental conditions (i.e. the musculoskeletal system is reduced to the force applied on the joint replacement being designed). ${ }^{30,31}$

b) Molecular dynamics simulations are used to explore the fundamental chemistry of new pharmaceutical compounds, for example in their interaction with the carrier. $^{32}$

c) Population pharmacokinetics are extensively used to investigate the absorption, distribution, metabolism, and excretion of new drugs. ${ }^{33}$

d) More recently, systems biology modelling has been used in discovery, and relation to specific risks such as cardiotoxicity. $^{34}$

Most of these uses are very early in the development cycle, around design/discovery activities, so they make very little impact on the cost of innovation, which is dominated by the trials costs. What industry needs are in silico technologies that help to reduce, refine or partially replace animal and human experimentation. This terminology, normally used in the context of animal experimentation reduction, can be applied also to humans with the following terminological clarification, to be used hereinafter:

a) Reduce means the reduction of the number of animals or humans involved in the experimentation, and/or the duration of such involvement.

b) Refine means the reduction of the suffering or of simply the risks the experimentation involves. However, here we will consider the reduction of suffering and risk in relation to how effectively the trial fulfills its purpose; that is, how accurately the trial predicts the efficacy and the safety of the new product once it becomes widely used. So, refinement can be achieved by reducing the suffering or risks associated with the trial while its usefulness remains unchanged, or improving its usefulness while keeping the suffering/risk unchanged.

c) Replacement refers to the possibility of avoiding the trial entirely. While this is a theoretical option for animal trials, ${ }^{35}$ it is not for human trials. Thus, we use the expression partial replacement.
Developing in silico technologies to reduce, refine and partially replace in vivo experimentation requires overcoming barriers of three kinds: knowledge, as we to some extent still lack the fundamental and technological knowledge required; reliability, enough to entrust part of a fundamentally mission-critical process to a new technology; and adoption, which involves changes in business practice that the adoption of disruptive technologies always involve. There are three actors central to the development of in silico technologies to reduce, refine and partially replace in vivo experimentation: the academic research community that should overcome the knowledge barriers, the regulators who should address the reliability barriers, and industry that should address the adoption barriers. But the industrial providers of modeling and simulation technologies largely do not work around biomedical products, and the industries developing biomedical products largely do not work with simulation technologies; researchers need industrial guidance to orient their efforts where is necessary, and regulators cannot develop reliability in silico technologies without the support of researchers and industry experts. Before any solid innovation in this area can appear, we need to create a community of practice, where all these stakeholders discuss and network, in order to produce a research and technological development roadmap to orient effectively all efforts.

\section{The process}

The European Commission funded the Avicenna support action with the specific purpose to elaborate a research and technological research roadmap for the area referred as In Silico Clinical Trials (ISCT). The action ran from October 2013 until September 2015; the process the Avicenna consortium used to develop this roadmap can be summarised in three steps.

\section{Form a community of practice;}

- Capture the consensus of the experts within this community.

- Consolidate all the inputs in a final draft version of the roadmap.

- Publicly validate the roadmap with all stakeholders.

The community of practice was formed starting from a nucleus of roughly 200 experts who expressed preliminary interest for the Avicenna action, which expanded during the duration of the consensus process to 577 experts. Over the 24 months of activity we contacted nearly 5,000 experts worldwide, informing them of the Avicenna action. Table 1 details the composition of this community of practice in terms of expertise. Researchers represented $29 \%$ of the experts' panel, followed by producers of biomedical products $(26 \%)$, providers of 
clinical trial services $(25 \%)$, regulators $(12 \%)$, and others $(8 \%)$. In terms of nationality $69 \%$ were from Europe, Europe, UK was the most represented state (17\%), followed by Italy (12\%), France, Germany and Spain (7\%); overall 37 countries were represented, including 25 of the $28 \mathrm{EU}$ member states. Most experts participated
$22 \%$ from USA, and $10 \%$ from other countries. Within

in the consensus process remotely, but 105 of them attended at least one of the five events the Avicenna action organised in Rome, Brussels, Lyon, and Barcelona.

Table 1: Number of experts engaged in the Avicenna consensus process, subdivided by their clusters and expertise.

\begin{tabular}{|c|c|c|c|c|c|c|c|}
\hline & Consortium & Consumers & Producers & Providers & Regulatory & Research & Spenders \\
\hline Consortium & 17 & & & & & & \\
\hline Patient's Orgs & & 6 & & & & & \\
\hline Charity & & 6 & & & & & \\
\hline Medical Devices & & & 78 & & & & \\
\hline Large Biopharma & & & 65 & & & & \\
\hline Small Biopharma & & & 9 & & & & \\
\hline ISCT & & & & 42 & & & \\
\hline Software \& hardware & & & & 39 & & & \\
\hline Consultants & & & & 26 & & & \\
\hline Research Hospitals & & & & 21 & & & \\
\hline $\mathrm{CRO}$ & & & & 9 & & & \\
\hline Trade organisation & & & & 6 & & & \\
\hline Data Banks & & & & 3 & & & \\
\hline National & & & & & 53 & & \\
\hline Supranational & & & & & 14 & & \\
\hline Math, physics, and en & sciences & & & & & 56 & \\
\hline Computational biome & & & & & & 55 & \\
\hline Medical Research & & & & & & 30 & \\
\hline social and economica & & & & & & 15 & \\
\hline Biology, systems biol & iochemistry & & & & & 12 & \\
\hline Funders & & & & & & & 12 \\
\hline Banking \& insurance & & & & & & & 3 \\
\hline Total $\mathrm{x}$ each cluster & 17 & 12 & 152 & 146 & 67 & 168 & 15 \\
\hline
\end{tabular}

To those experts who agreed to get involved in the process, we offered an opt-out mechanism that allowed them the option of removing themselves at any time if they did not agree with the consensus process. Very few took this option during the process and the numbers reported here refer to those who agreed to sign the final roadmap, and whose names are listed in its appendix.

The consensus process over such a wide and heterogeneous community required a specialised approach. In 2005, Thomas Schelling received the Nobel Prize in Economics for having enhanced our understanding of conflict and cooperation through gametheory analysis. In particular, he developed the concept of a focal point (known as a Schelling point), which is the solution to an opportunity most people will select when sub-optimal communication hinders consensus building. From this, and two related behavioural sciences, Alignment Optimisation (AO) has emerged as a management science, providing a crowd-sourcing knowledge discovery process that efficiently yields endorsed, coordinated actions for a group with a shared purpose. $\mathrm{AO}$, in the on-line implementation provided by SchellingPoint, was selected as the primary method for crowd-sourcing knowledge from participants in the Avicenna process, based on previous experience of one of the partners using the technology in a similar context relating to the application of systems biology to drug discovery and development. ${ }^{36}$ Overall 214 experts actively participated in the $\mathrm{AO}$ consensus process. In addition, five physical meetings where organised; in four the participation was capped to 50 delegates to ensure an effective interactions. The fifth was a public event aimed to validate broadly the final draft of the roadmap.

Initially the roadmap was intended to be a single booklet to be read in its entirety by all stakeholders. Thus, we organised a first tentative index for such a document, and started to populate it with the inputs generated by the AO process. At each cycle a stand-alone document or 'position paper' was derived from the current draft, and 
circulated to all experts in advance of the meeting. Written comments, as well as all the inputs collected during the meeting were combined with the outputs of the following AO cycle to compose the next draft. After the third event in October 2014, the complexity of the roadmap started to increase exponentially. New sections were added, some of which were relevant only to some stakeholders. During the first review meeting with the European Commission, the reviewers identified the need for a structured approach, a sort of reading guide that would point each category of stakeholder to read only those chapters that were relevant to them. As a result of these reflections and after the fourth event, the roadmap was completely re-organised. The document was divided into 11 chapters, each one designed to be readable either as a stand-alone document, or together with the others. We developed a reading guide for different categories of readers to ensure an effective comprehension of the roadmap. After this reorganisation, a draft version of each chapter was posted as an unformatted google doc open for editing to anyone with the link. The links were sent to all members of the avicenna community of practice, giving everyone the opportunity to edit the content of the entire roadmap. In parallel, a Mendeley bibliographic database, also public, was made available for everyone to add relevant papers to be cited in the roadmap. After this revision round, the text was collected, and formatted into Microsoft Word documents, with the inclusion of figures and bibliographic references. The resulting document was posted on the public avicenna website and all the available communication channels were used to invite our experts, as well as any other interested parties to revise and comment on these documents. The final draft roadmap was circulated in advance of the final Avicenna meeting, where it was discussed extensively. All comments collected online or during Event Five were consolidated into the final version of the roadmap, which can be freely downloaded in PDF format from Research Gate. The list of experts involved in the consensus process can be found in Annex 1 of the roadmap. ${ }^{1}$

The full roadmap, with its 116 pages is massive document. Here we will try to summarise some of the key elements: the definition of ISCT, the use cases that emerged, the challenges that remain to be met for a full adoption, and some of the key recommendations to funders and policy makers.

\section{Definition of ISCT}

A lot of time was spent in discussing a definition of in silico clinical trials that was broad enough to cover all relevant use cases, but specific enough to be informative and useful. Consensus was reached on the following:

The use of individualised computer simulation in the development or regulatory evaluation of a medicinal product, medical device, or medical intervention. It is a subdomain of 'in silico medicine', the discipline that encompasses the use of individualised computer simulations in all aspects of the prevention, diagnosis, prognostic assessment, and treatment of disease.

Two elements are worthy of further explanation: firstly, the reference to individualised computer simulations and, secondly, references to biomedical products in general.

The choice of the word individualised in place of the more popular personalised reflects a majority view amongst our experts that the latter is primarily used to indicate stratification by genetic information, whereas here we refer to the use of all available information including genomics, post-genomics, metabolomics, microbiomics, medical imaging, biomedical instrumentation (including implanted, wearable, or environmental sensors), self-reported, and clinical data. The roadmap identifies three levels of individualisation, which are defined in terms of how the predictive accuracy of the model is quantified:

Level 1: The model intends to represent one generic individual of the reference population. Its predictive accuracy is measured by comparing the model predictions to the range of observed values in the reference population.

Level 2: The model intends to represent an average individual of the reference population. Its predictive accuracy is measured by comparing the model predictions to the central properties (average, median, etc.) of distribution of values observed in the reference populations.

Level 3: The model intends to represent each individual of the reference population. Its predictive accuracy is measured by comparing the predicted values to the observed values for each individual in the reference population.

Regarding the decision to refer generically to biomedical products, in spite of the profound difference that exists between pharmaceutical products and medical devices, our experts agreed that there are lessons to be learnt across these industrial sectors, although many aspects are specific. As a result the roadmap includes in depth sections specifically for drugs and devices, and others that are more general.

\section{In silico clinical trials: use cases current and future}

At the cost of oversimplifying, we can reduce the development and assessment cycle of a biomedical product into three macro-phases: design/discovery, preclinical assessment, clinical assessment; in silico technologies have the potential to impact positively on all three. While the Avicenna roadmap addresses all three phases, here we will focus only on the potential use of the use of individualised computer models in the reduction, refinement, and partial replacement of animal and human experimentation. 
A first use case that emerged in the consensus process is that where individualised computer models are used in combination with imaging and sensing to produce surrogate measurements for biomarkers that cannot be directly measured non-invasively, but whose longitudinal evolution is highly informative of the efficacy or of the safety of the product being tested. This can be applied both in pre-clinical studies on animals, and in clinical studies on humans. For example in vivo computed tomography data can inform individualised finite element models that accurately predict non-invasively the changes in bone strength or joint stresses in longitudinal studies, both in mice, ${ }^{37-40}$ and in humans. ${ }^{41-45}$ This use case is related to refinement, in the definition we provided above, as modelling can replace invasive measurements that involve suffering in animals, and increased risk in humans; or improving the usefulness of the in vivo trials by making measurable a more significant biomarker, while retaining the same level of risk/suffering.

A second is the use of individualised models to reduce the number of animals or humans involved in the experimentation, and/or the duration of such involvement. A first example is related to the previous use case: when a surrogate measurement considerably improves the experimental reproducibility of the in vivo study, the numbers required for statistical significance is reduced. A second example is when the endpoint can be surrogated by individualised model prediction: for example a clinical trial of a new bone drug aimed to preserve the mechanical strength of bones as a means to avoid bone fractures could replace the fracture endpoint, with a bone strength endpoint, which is highly predictive of the risk of bone fracture. ${ }^{43}$

A third is where individualised models can partially replace animals or humans in a trial. The USA Food and Drug Administration (FDA) has already approved the complete replacement of experiments on dogs with a diabetes type I simulator 35 for the de-risking of new artificial pancreas technologies. In silico tools are being considered as possible replacements for animal experimentation in the pre-clinical assessment of cardiotoxicity. ${ }^{46,47}$ For humans the argument is more complex as all experts exclude the complete replacement of human clinical trials for high-risk products, in the foreseeable future. However, a possible scenario is that of using individualised models to generate virtual patients with specific characteristics to supplement clinical trials on real patients. One possible approach is to design the trial using a Bayesian approach, and to treat the predictions from the virtual patients as an a priori probability. $^{48}$

A fourth somehow related use case refers to situations where the de-risking with conventional clinical trials is impossible, or unsustainable. Osteogenesis imperfecta (OI) has a prevalence of 1/20,000 new born, which makes roughly 200 children per year in the USA. A phase III clinical trial that would involve 2000 OI patients of the same age would be impossible. But the problem is broader than this; if a severe complication with a new drug appears only in patients with a combination of traits that appear in the general population with a probability of one in 10,000, such a complication could never appear in any clinical trial. However, the severity of the complication might make it still a concern, even with such low probability when the product is commercialised. This problem is particularly acute in medical devices, where it is not uncommon to obtain pre-market approval on the basis of trials involving only one or two hundred patients. In all these cases in silico-augmented clinical trials can be designed to use virtual patients to explore less common phenotypes (anatomical, functional, metabolic, or genetic) that by random sampling would be quite difficult to recruit. Of course this can in principle be done also experimentally, but creating a virtual patient with a BMI $>90$ is surely much easier than recruiting one, for example.

\section{The challenges}

As we wrote in the introduction, developing in silico technologies to reduce, refine and partially replace in vivo experimentation requires overcoming knowledge, reliability, and adoption barriers. The Avicenna roadmap extensively analyses all these barriers, developing a broad research and technological roadmap that research-funding agencies should use to orient their investments in this area. Here, again for brevity, we mention only a few challenges that were considered by our experts to be of greater importance.

\section{Reducing, refining, and partially replacing clinical trials}

Further research is required on the use of in silico technologies for the reduction, refinement, and partial re placement of product trials on animals and on humans. Research is required into methods through which subjectspecific models can provide more reproducible outcome measures, reducing the size of the animal or human cohorts required for statistical significance. Trial refinement using subject-specific models replacing where possible animals' vivisection and reducing risk in human trials should also be examined. Additionally, studies are required to examine the ways in which subject-specific models might partially replace trials, by augmenting or complementing the in vivo cases with in silico versions. Finally, research should be conducted into how subjectspecific models can transform product trials targeting paediatric and rare diseases.

We also recommend research towards the development and validation of in silico models that assist in scaling and extrapolating observations from in vitro and in vivo studies on animals into possible outcomes in vivo in humans. 


\section{The physiological envelope, the deployment envelope}

The entire range of possible values a physiological parameter can assume in a given subject during daily life is referred as the physiological envelope. ${ }^{49}$ The Avicenna experts reached ample consensus that for the successful adoption of in silico clinical trial technologies, it is essential these technologies are also capable of individualising behavioural aspects such as nutrition, physical activity, consumption of alcohol or recreational drugs, constipation, therapeutic compliance, etc. In other words it is essential to develop the methods and the technologies that allow the definition of a reliable physiological envelope for each physiological biomarker that characterises those behaviours that could in any way interfere or affect the biomedical product under assessment. For products that are deployed (i.e. surgically implanted medical devices), a similar reason applies to the deployment envelop, i.e. the quantification of the reproducibility of the deployment/implantation of specific classes of biomedical products.

\section{From validation to confidence}

Another grand challenge for in silico clinical trials is the establishment of sufficient confidence, among practitioners and regulators. This is usually reduced to the specific aspects of verification, validation and uncertainty quantification as formulated for models used in other industrial contexts. But there is a need for a framework that specifically addresses the needs of the biomedical industry. In January 2014 the FDA produced draft guidance for staff and industry on "Reporting of Computational Modeling Studies in Medical Device Submissions. Then they contributed to the establishment, within the American Society of Mechanical Engineering standardisation committees, of a Verification and Validation V and V-40 sub-committee charged of developing a new technical standard for the verification and validation in computational modelling of medical devices.

But the problem is broader, and involves three major barriers:

- Cultural resistance from the specialist workforce, mostly formed by biologists, pharmacologists, and medical practitioners with a limited background in mathematics and physics.

- The resistance of regulators, who historically have not accepted evidence obtained in silico for the certification process of new biomedical products, especially those in higher risk categories; and

- The inherent complexity associated with the accurate, quantitative modelling of living organisms. There are signals that suggest this situation is changing, although probably not as quickly as it could.
A necessary step is the activation of research projects aimed at demonstrating the reliability of ISCT, for example by comparing retrospectively the results obtained in silico with those obtained in vivo.

We also recommend methodological research on frameworks for the assessment of the predictive accuracy of subject-specific models when the quantities to be predicted can be observed experimentally only with large uncertainty.

\section{Automation for high-throughput}

Currently the vast majority of subject-specific modelling techniques require significant amounts of manual work by an extremely specialised workforce. While this is acceptable in research settings, if these methods are to be adopted by the biomedical industry, the development of new high-throughput methods automating subjectspecific modelling, including treatment simulation, is needed. This involves the large-scale execution of complex personalised simulations on substantial numbers of cases $(>1000)$, technologies that automate the replication of the ISCT if the product is modified, and visual analytics technologies to explore these data sets effectively, including interactive visualisation technologies that facilitate communication with nontechnical members of the product team.

\section{Recommendations}

The Avicenna roadmap includes 36 specific recommendations for various stakeholders, including educators, researchers, funders, policy makers, providers of ISCT technologies, etc. Here we report only a few, relevant for producers (i.e. biomedical industry), and regulators.

R23 - We recommend that the producers of biomedical products contribute to precompetitive collaborations with the aim of:

a. Establishing an in silico assessment framework for each family of devices, which investigates all relevant failure modes for that device. This will enable research groups to extend the framework with refined/alternative predictors for the various failure modes.

b. Evaluating retrospectively a number of biomedical products, both successful and unsuccessful, for which the clinical outcome is well known, in order to build confidence in the methods.

c. Running double-blind ISCT in parallel with existing in vivo clinical trials, comparing the current best practice with modified approaches that include modelling and simulation. Precedence should be given to critical areas such as paediatric and rare diseases, drug retargeting, etc. 
R29 - We recommend that regulatory bodies across the world embrace the innovation offered by in silico technologies and, in collaboration with academic and industrial experts, develop the framework of standards, protocols, and shared resources required to evaluate the safety and the efficacy of biomedical products using ISCT technologies.

$\mathrm{R} 30$ - We recommend that regulators consider also regulating in silico services to be used for the assessment of biomedical products as medical devices in their own right (i.e. software as a medical device).

R31 - We recommend that all European regulators follow the approach used by the USA Food and Drug Administration, which has recognised the strategic potential of in silico technologies, such as with the Medical Device Development Tool process and is working closely and publicly with academia and industry to develop standards and protocols that ensure their effective and safe adoption. We endorse the formation of the Medical Devices Innovation Consortium, as a vehicle for such pre-competitive collaborative efforts.

R32 - As the Avicenna consensus process has demonstrated, in a globalised economy the discourse on ISCT must develop worldwide; thus, we recommend that all agencies remove as many barriers as possible, and actively support pre-competitive research and technological development across countries and continents.

R35 - We recommend that the Avicenna Community of Practice, and in particular the industrial and academic stakeholders, form the Avicenna Alliance for Predictive Medicine, to coordinate and implement public and privately funded research on this topic, and to develop policy recommendations designed to overcome all barriers that slow or limit the adoption of ISCT technologies. This alliance should work closely with the Innovative Medicines Initiative public-private partnership in order to avoid duplication and favour synergy.

In Silico Clinical Trials can truly transform the biomedical industry, and they represent the best hope for the long-term sustainability of universal models of healthcare. The time is now, the challenge is huge; and only if we all work together will we succeed.

\section{ACKNOWLEDGEMENTS}

The authors would like to thank Karen El-Arifi, Martina Contin, Anders Karlström, Mike Taylor, and Callum MCgregor for the support in running the Avicenna action, and all 577 experts listed in Annex 1 of the Avicenna Roadmap, without whom nothing of this would have been possible.
Funding: The European Commission as part of the $7^{\text {th }}$ Framework Program for Research and Technological Development (FP7). Project reference: 611819.

Conflict of interest: None declared

Ethical approval: Not required

\section{REFERENCES}

1. Viceconti M, Henney A, Morley-Fletcher E, eds. In silico Clinical Trials: how computer simulation will transform the biomedical industry. Brussels: Avicenna Consortium 2016. DOI: 10.13140/RG.2.1.2756.6164

2. Solomon AK, Gold GL. Potassium transport in human erythrocytes: evidence for a three compartment system. J Gen Physiol. 1955;38:37188. Available http://jgp.rupress.org/content/38/3/371.full.pdf.

3. Eberl S, Anayat AR, Fulton RR, Hooper PK, Fulham MJ. Evaluation of two population-based input functions for quantitative neurological FDG PET studies. European Journal Of Nuclear Medicine. 1997;24:299-304.

4. Bouxsein ML, Melton LJ, Riggs BL. Age and sexspecific differences in the factor of risk for vertebral fracture: a population-based study using QCT. Journal of bone and mineral research: J Bone Miner Res. 2006;21:1475-82.

5. Chabaud S, Girard P, Nony $P$ and Boissel JP. Clinical trial simulation using therapeutic effect modeling: application to ivabradine efficacy in patients with angina pectoris. J Pharmacokinet Pharmacodyn. 2002;29:339-63.

6. Clermont G, Bartels J, Kumar R, Constantine G, Vodovotz Y, Chow C. In silico design of clinical trials: a method coming of age. Critical Care Medicine. 2004;32:2061-70.

7. Duval V, Chabaud S, Girard P, Cucherat M, Hommel M, Boissel JP. Physiologically based model of acute ischemic stroke. Journal of cerebral blood flow and metabolism. J Cereb Blood Flow Metab. 2002; 22: 1010-8.

8. Kansal AR,Trimmer J. Application of predictive biosimulation within pharmaceutical clinical development: examples of significance for translational medicine and clinical trial design. Systems Biology. 2005;152:214-20.

9. Ribba B, Saut O, Colin T, Bresch D, Grenier E, Boissel JP. A multiscale mathematical model of avascular tumor growth to investigate the therapeutic benefit of anti-invasive agents. Journal of Theoretical Biology. 2006;243:532-41. Available at http://ac.els-cdn.com/S0022519306003043/1s2.0-S0022519306003043-main.pdf?_tid=ef5886a4f6f0-11e4-99c2-

00000aacb360\&acdnat=1431247719_9e4e61f92a12 bf8e4c69084e644382e5.

10. Rostami-Hodjegan A, Tucker GT. Simulation and prediction of in vivo drug metabolism in human 
populations from in vitro data. Nature Reviews Drug Discovery. 2007;6:140-8.

11. Lomnicki A. Individual-based Models in Population Ecology. eLS. John Wiley \& Sons, Ltd, 2001. Available at http://onlinelibrary.wiley.com/doi/10.1002/9780470 015902.a0003312.pub2/abstract.

12. Chabanas M, Luboz V and Payan Y. Patient specific finite element model of the face soft tissues for computer-assisted maxillofacial surgery. Medical Image Analysis. 2003;7:131-51.

13. Fernandez JW, Hunter PJ. An anatomically based patient-specific finite element model of patella articulation: towards a diagnostic tool. Biomechanics and Modeling In Mechanobiology. 2005;4:20-38.

14. Li NYK, Verdolini K, Clermont G. A patientspecific in silico model of inflammation and healing tested in acute vocal fold injury. PloS one. 2008;3: e2789. Available at http://www.ncbi.nlm.nih.gov/pmc/articles/PMC248 1293/pdf/pone.0002789.pdf.

15. O'Rourke MJ, McCullough JP. A comparison of the measured and predicted flowfield in a patientspecific model of an abdominal aortic aneurysm. Proceedings of the Institution of Mechanical Engineers Part H, Journal of Engineering in Medicine. 2008;222:737-50. Available at http://pih.sagepub.com/content/222/5/737.full.pdf.

16. Viceconti M, Pancanti a, Dotti M, Traina F, Cristofolini L. Effect of the initial implant fitting on the predicted secondary stability of a cementless stem. Medical \& Biological Engineering \& Computing. 2004;42:222-9.

17. Wolters BJBM, Rutten MCM, Schurink GWH, Kose U, Hart JD, Vosse FN VD. A patient-specific computational model of fluid-structure interaction in abdominal aortic aneurysms. Medical Engineering \& Physics. 2005;27:871-83.

18. PriceWaterhouseCoopers. Pharma 2020: Virtual R \& D Which path will you take ? 2008.

19. Petrini L, Trotta A, Dordoni E. A Computational Approach for the Prediction of Fatigue Behaviour in Peripheral Stents: Application to a Clinical Case. Ann Biomed Eng. 2015. Available at http://www.ncbi.nlm.nih.gov/pubmed/26433586

20. Hulme PA, Boyd SK, Heini PF, Ferguson SJ. Differences in endplate deformation of the adjacent and augmented vertebra following cement augmentation. Eur Spine J. 2009;18:614-23. Available http://www.ncbi.nlm.nih.gov/pubmed/19242738

21. Available http://www.ncbi.nlm.nih.gov/pmc/articles/PMC323 4009/pdf/586_2009_Article_910.pdf.

22. Corrias A, Jie X, Romero L, M. J. Bishop,1 M. Bernabeu,1 E. Pueyo. Arrhythmic risk biomarkers for the assessment of drug cardiotoxicity: from experiments to computer simulations. Philos Trans
A Math Phys Eng Sci. 2010;368:3001-25. Available at http://www.ncbi.nlm.nih.gov/pubmed/20478918

23. Dronne MA, Grenier E, Dumont T, Hommel M, Boissel JP. Role of astrocytes in grey matter during stroke: a modelling approach. Brain Research. 2007; 1138:231-42. Available at http://ac.elscdn.com/S0006899306036845/1-s2.0-

S0006899306036845-main.pdf?_tid=fb6766fe-f6f011e4-a3ba-

00000aab0f01\&acdnat=1431247739_eb246a0b9749 5ee32d9bf68008f80a58

24. Bousquet J, Jorgensen C, Dauzat M. Systems medicine approaches for the definition of complex phenotypes in chronic diseases and ageing. From concept to implementation and policies. Current Pharmaceutical Design. 2014;20:5928-44.

25. Wolkenhauer O, Auffray C, Brass O, Clairambault J, Deutsch A, Drasdo D. Enabling multiscale modeling in systems medicine. Genome Medicine. 2014;6:21. Available at http://www.ncbi.nlm.nih.gov/pmc/articles/PMC406 2045/pdf/gm538.pdf.

26. Wang RS, Maron BA, Loscalzo J. Systems medicine: evolution of systems biology from bench to bedside. Wiley Interdiscip Rev Syst Biol Med. $2015 . \quad$ Available http://www.ncbi.nlm.nih.gov/pubmed/25891169

27. Hood L, Balling R, Auffray C. Revolutionizing medicine in the $21^{\text {st }}$ century through systems approaches. Biotechnology Journal. 2012;7:9921001.

28. Available http://www.ncbi.nlm.nih.gov/pmc/articles/PMC396 2497/pdf/nihms-525368.pdf.

29. Noble D. The future: putting Humpty-Dumpty together again. Biochem Soc Trans. 2003;31:156-8. Available http://www.ncbi.nlm.nih.gov/pubmed/12546675

30. Boissel JP, Kahoul R, Marin D, Boissel FH. Effect model law: an approach for the implementation of personalized medicine. J Pers Med. 2013;3:177-90. Available http://www.ncbi.nlm.nih.gov/pubmed/25562651

31. Boissel JP, Auffray C, Noble D, Hood L, Boissel F. Bridging Systems Medicine and Patient Needs. CPT: Pharmacometrics \& Systems Pharmacology. Wiley-Blackwell, 2015.

32. Viceconti M, Cristofolini L, Toni A. Design revision of a partially cemented hip stem. Proc Inst Mech Eng H. 2001;215:471-8. Available at http://www.ncbi.nlm.nih.gov/pubmed/11726047

33. Hose DR, Narracott AJ, Penrose JM, Baguley D, Jones IP, Lawford PV. Fundamental mechanics of aortic heart valve closure. J Biomech. 2006;39:958$67 . \quad$ Available http://www.ncbi.nlm.nih.gov/pubmed/16488234.

34. Eslami M, Nikkhah SJ, Hashemianzadeh SM, Sajadi SA. The compatibility of tacrine molecule with poly (n-butylcyanoacrylate) and chitosan as efficient carriers for drug delivery: a molecular dynamics 
study. Eur J Pharm Sci. 2016;82:79-85. Available at http://www.ncbi.nlm.nih.gov/pubmed/26598087.

35. Mungall DR, Ludden TM, Marshall J, Hawkins DW, Talbert RL, Crawford MH. Population pharmacokinetics of racemic warfarin in adult patients. J Pharmacokinet Biopharm. 1985;13:21327. Available http://www.ncbi.nlm.nih.gov/pubmed/3841364.

36. Rodriguez B, Burrage K, Gavaghan D, Grau V, Kohl P, Noble D. The systems biology approach to drug development: application to toxicity assessment of cardiac drugs. Clin Pharmacol. 2010;88(1):130-4.

37. Visentin R, Dalla Man C, Kovatchev B, Cobelli C. The university of Virginia/Padova type 1 diabetes simulator matches the glucose traces of a clinical trial. Diabetes Technol Ther. 2014;16:428-34. Available http://www.ncbi.nlm.nih.gov/pubmed/24571584

38. Henney A, Superti-Furga G. A network solution. Nature. 2008;455:730-1. Available at http://www.nature.com/nature/journal/v455/n7214/p df/455730a.pdf.

39. Lu Y, Boudiffa M, Dall'Ara E, Bellantuono I,Viceconti M. Evaluation of in-vivo measurement errors associated with micro-computed tomography scans by means of the bone surface distance approach. Med Eng Phys. 2015;37:1091-7. Available at http://www.ncbi.nlm.nih.gov/pubmed/26427546.

40. Nyman JS, Uppuganti S, Makowski AJ, Rowland BJ, Merkel AR, Sterling JA. Predicting mouse vertebra strength with micro-computed tomographyderived finite element analysis. Bonekey Rep. 2015; $4: 664$. Available http://www.ncbi.nlm.nih.gov/pubmed/25908967.

41. Pate KM, Sherk VD, Carpenter RD, Weaver M, Crapo S, Gally F. The beneficial effects of exercise on cartilage are lost in mice with reduced levels of ECSOD in tissues. J Appl Physiol(1985). 2015;118:760-7. Available http://www.ncbi.nlm.nih.gov/pubmed/25593283.

42. Borges P DN, Forte AE, Vincent TL, Dini D and Marenzana M. Rapid, automated imaging of mouse articular cartilage by micro CT for early detection of osteoarthritis and finite element modelling of joint mechanics. Osteoarthritis Cartilage. 2014;22:141928.

http://www.ncbi.nlm.nih.gov/pubmed/25278053.

43. Orwoll ES, Marshall LM, Nielson CM, Cummings SR, Lapidus J, Cauley JA. Finite element analysis of the proximal femur and hip fracture risk in older men. J Bone Miner Res. 2009;24:475-83. Available at http://www.ncbi.nlm.nih.gov/pubmed/19049327.
44. Kopperdahl DL, Aspelund T, Hoffmann PF, Sigurdsson S, Siggeirsdottir K, Harris TB. Assessment of incident spine and hip fractures in women and men using finite element analysis of CT scans. J Bone Miner Res. 2014;29:570-80. Available http://www.ncbi.nlm.nih.gov/pubmed/23956027.

45. Falcinelli C, Schileo E, Balistreri L, Baruffaldi F, Bordini B, Viceconti M, et al. Multiple loading conditions analysis can improve the association between finite element bone strength estimates and proximal femur fractures: A preliminary study in elderly women. Bone. 2014;71-80. Available at http://www.ncbi.nlm.nih.gov/pubmed/25014885.

46. Qasim M, Farinella G, Zhang J, Li X, Yang L, Eastell R, Viceconti M. Patient-Specific Finite Element Minimum Physiological Strength as Predictor of the Risk of Hip Fracture: The effect of methodological determinants. Osteoporosis International. 2016;1-8. DOI: 10.1007/s00198-0163597-4

47. Li X, Viceconti M, Cohen MC, Reilly GC, Carre MJ, Offiah AC. Developing CT based computational models of pediatric femurs. J Biomech. 2015;48:2034-40. Available at http://www.ncbi.nlm.nih.gov/pubmed/25895643.

48. Yuan Y, Bai X, Luo C, Wang K and Zhang H. The virtual heart as a platform for screening drug cardiotoxicity. Br J Pharmacol. 2015;172:5531-47. Available http://www.ncbi.nlm.nih.gov/pubmed/25363597.

49. Zemzemi N, Bernabeu MO, Saiz J, Cooper J, Pathmanathan P, Mirams GR, Francis JP. Computational assessment of drug-induced effects on the electrocardiogram: from ion channel to body surface potentials. Br J Pharmacol. 2013;168:718$33 . \quad$ Available at http://www.ncbi.nlm.nih.gov/pubmed/22946617.

50. Haddad T, Himes A, Campbell M. Fracture prediction of cardiac lead medical devices using Bayesian networks. Reliability engineering \& system safety. 2014;123:145-57. Available at http://www.sciencedirect.com/science/article/pii/S0 951832013003025.

51. Viceconti M, Hunter P, Hose R. Big data, big knowledge: big data for personalized healthcare. IEEE J Biomed Health Inform. 2015;19:1209-15. Available at http://www.ncbi.nlm.nih.gov/ pubmed/26218867.

Cite this article as: Viceconti M, Henney A, MorleyFletcher E. In silico clinical trials: how computer simulation will transform the biomedical industry. Int J Clin Trials 2016;3(2):37-46. 